\title{
Ultrarelativistic bound states in spinor and scalar QED
}

\author{
William Dykshoorn and Roman Koniuk \\ Department of Physics, York University, Toronto, Ontario, Canada M3J 1 P3 \\ Ramon Muñoz-Tapia \\ Departament de Fisica Térica, 08193 Bellaterra, Barcelona, Spain
}

(Received 22 August 1989)

\begin{abstract}
Nonperturbative integral equations are developed variationally for two-particle bound states in spinor and scalar quantum electrodynamics. The equations are solved numerically in the massive case and analytically in the massless case. The coupling constant is varied in order to study the bound states in the ultrarelativistic region.
\end{abstract}

\section{INTRODUCTION}

Producing the hadron spectrum from quantum chromodynamics (QCD) has proven to be a formidable task indeed. This is essentially because the low-energy modes in the theory couple strongly, making the problem inaccessible to perturbation theory. The strong-coupling problem can be divided (somewhat artificially) into two aspects. In a Fock-space expansion of the states, strong coupling will imply large mixings and slow convergence to the physical states. Even within a limited sector of Fock space, relativistic effects will be large and perturbation theory will not be useful.

Although the variational technique, ${ }^{1}$ which we advocate in this paper, is in principle suitable to attacking both aspects, it is the latter, more modest aspect of the problem to which we address ourselves at least as a first step.

To this end we study spinor and scalar quantum electrodynamics (QED). (In a limited Fock space only the Abelian part of QCD survives). ${ }^{2}$

In Sec. II we present the respective Hamiltonians and variationally derive the corresponding integral equations for the bound-state wave functions. In Sec. III we present our numerical method and results. Comparisons with analytic results of the massless theory are made in Sec. IV along with our concluding remarks.

\section{HAMILTONIANS}

\section{A. Spinor QED}

In radiation gauge the QED Hamiltonian is

$$
\begin{gathered}
H=\int d^{3} x\left(\psi^{\dagger}\{\boldsymbol{\alpha} \cdot[(1 / i) \boldsymbol{\nabla}-e \mathbf{A}]+\beta m\} \psi\right. \\
\left.+\frac{1}{2}\left(\mathbf{E}^{2}+\mathbf{B}^{2}\right)\right),
\end{gathered}
$$

where

$$
\begin{aligned}
& \mathbf{E}^{2}=\mathbf{E}_{t}^{2}+\mathbf{E}_{l}^{2}, \quad \mathbf{E}_{t}=-\dot{\mathbf{A}}, \quad \mathbf{B}=\boldsymbol{\nabla} \times \mathbf{E}, \\
& \mathbf{E}_{l}^{2}=\frac{e^{2}}{4 \pi} \int d^{3} y \frac{\psi^{\dagger}(\mathbf{x}) \psi(\mathbf{x}) \psi^{\dagger}(\mathbf{y}) \psi(\mathbf{y})}{|\mathbf{x}-\mathbf{y}|} .
\end{aligned}
$$

We construct an ansatz for the fermion-antifermion bound state by acting on the vacuum with a bilocal operator folded against a probability amplitude, namely

$\left|e^{+} e^{-}\right\rangle=\int d^{3} x d^{3} y f(|\mathbf{x}-\mathbf{y}|): \bar{\psi}(\mathbf{x}) \Gamma \psi(\mathbf{y}):|0\rangle$

Expanding the field operators in the standard way, this expression Fourier transforms itself and becomes

$$
\begin{array}{r}
\left|e^{+} e^{-}\right\rangle=\int d^{3} p \sum_{s s^{\prime}} \widetilde{f}(p) \bar{u}(\mathbf{p}, s) \Gamma v\left(-\mathbf{p}, s^{\prime}\right) \\
\times b^{\dagger}(\mathbf{p}, s) d^{\dagger}\left(-\mathbf{p}, s^{\prime}\right)|0\rangle,
\end{array}
$$

where $\widetilde{f}(p)$ is the momentum-space wave function depending only on magnitude $p$. The angular and spin information is contained in the spinor factor with the matrix $\Gamma$ chosen to be $\gamma_{5}, 1, \gamma, \gamma_{5} \gamma$, depending on whether we wish to construct a pseudoscalar, scalar, vector, or pseudovector state, respectively. The expectation value of the Hamiltonian is now calculated. We normal order the theory so that we can read off the bound-state mass directly since the Hamiltonian listed in (1) with ansatz (2) will give rise to Coulomb self-energy mass renormalization. Thus we calculate

$$
M[f(p)]=\frac{\left\langle e^{+} e^{-}|H| e^{+} e^{-}\right\rangle}{\left\langle e^{+} e^{-} \mid e^{+} e^{-}\right\rangle}
$$

which we variationally optimize with respect to $\widetilde{f}(p)$. This leads to the integral equation

$$
\begin{aligned}
& \left(2 E_{p}-M\right) \operatorname{Tr}\left[(\not p+m) \Gamma\left(\not p^{\dagger}-m\right) \bar{\Gamma}\right] \tilde{f}(p), \\
& \left.\frac{-\alpha}{8 \pi^{2}} \int \frac{d^{3} q}{E_{p} E_{q}} \frac{1}{|\mathbf{p}-\mathbf{q}|^{2}}\left[\operatorname{Tr}(\not p+m) \Gamma(\not)^{\dagger}-m\right)(\not-m) \Gamma^{\dagger}\left(\not^{\dagger}+m\right)\right] \tilde{f}(q)=0 .
\end{aligned}
$$


The traces and angular integration are now performed for the cases considered here and we obtain one-dimensional integral equations of the form

$$
M f(p)=2 E_{p} f(p)-\frac{\alpha}{4 \pi} \int \frac{q}{p} K(p, q) f(q) d q,
$$

where $M$ is the bound-state eigenvalue. The kernels are

$$
\begin{aligned}
0^{-+}: K(p, q)= & \frac{\left(E_{p}+E_{q}\right)^{2}}{E_{p} E_{q}} \ln \left|\frac{p+q}{p-q}\right|-\frac{2 p q}{E_{p} E_{q}} \text { (Ref. 3), } \\
0^{++}: K(p, q)= & \frac{\left(E_{p}+E_{q}\right)^{2}\left(E_{p} E_{q}-m^{2}\right)}{p q E_{p} E_{q}} \ln \left|\frac{p+q}{p-q}\right|-2-\frac{2 m^{2}}{E_{p} E_{q}}, \\
1^{--}: K(p, q)= & \frac{2 p q}{\left[\left(2 E_{p}^{2}+m^{2}\right)\left(2 E_{q}^{2}+m^{2}\right)\right]^{1 / 2}} \\
& \left.\times\left|\frac{\left(E_{p}+E_{q}\right)^{2}\left[\left(E_{p}+E_{q}\right)^{2}+2 m^{2}\right]}{4 p q E_{p} E_{q}} \ln \right| \frac{p+q}{p-q} \mid-2-\frac{E_{p}}{2 E_{q}}-\frac{E_{q}}{2 E_{p}}\right), \\
1^{++}: K(p, q)= & \frac{\left(E_{p}+E_{q}\right)^{2}\left[\left(E_{p}+E_{q}\right)^{2}-4 m^{2}\right]}{4 p q E_{p} E_{q}} \ln \left|\frac{p+q}{p-q}\right|-2-\frac{E_{p}}{2 E_{q}}-\frac{E_{q}}{2 E_{p}}-\frac{m^{2}}{E_{p} E_{q}} .
\end{aligned}
$$

\section{B. Scalar QED}

Proceeding as in the previous section the SQED Hamiltonian in radiation gauge is

$$
\begin{aligned}
& H=\int d^{3} x[ \pi^{*} \pi+(\boldsymbol{\nabla}+i e \mathbf{A}) \phi^{*} \cdot(\boldsymbol{\nabla}-i e \mathbf{A}) \phi \\
&\left.+m^{2} \phi^{*} \phi+\lambda\left(\phi^{*} \phi\right)^{2}+\frac{1}{2}\left(\mathbf{E}^{2}+\mathbf{B}^{2}\right)\right]
\end{aligned}
$$

where

$$
\begin{aligned}
& \mathbf{E}^{2}=\mathbf{E}_{t}^{2}+\mathbf{E}_{l}^{2}, \quad \mathbf{E}_{t}=-\dot{\mathbf{A}}, \quad \mathbf{B}=\boldsymbol{\nabla} \times \mathbf{A}, \\
& \mathbf{E}_{l}^{2}=+\int \frac{\rho(x) \rho(y)}{|\mathbf{x}-\mathbf{y}|} d^{3} y, \quad \rho(x)=i\left(\pi^{*} \phi-\phi^{*} \pi\right) .
\end{aligned}
$$

Our two-particle scalar ansatz is

$$
\left|\phi^{+} \phi^{-}\right\rangle=\int d^{3} x d^{3} y f(|\mathbf{x}-\mathbf{y}|): \phi^{*}(\mathbf{x}) \phi(\mathbf{y}):|0\rangle .
$$

This leads, as before, by variational optimization, to an integral equation of the form (6). We find that to obtain solutions for all values of $\alpha$, the value of $\lambda$ cannot be left arbitrary. This is due to the lack of convergence in the ultraviolet region of the integration. ${ }^{2}$ The singular behavior is removed by fixing $\lambda=\pi \alpha$.

In fact, at this value of $\lambda$ the integral equation is identical to the spinor pseudoscalar equation (7a) [after a suitable redefinition of $\widetilde{f}(p)$ ].

With all the integral equations now in place we can proceed to their solution.

\section{NUMERICAL METHOD AND RESULTS}

We use two methods to calculate the eigenvalues for the kernels listed in the previous section. In the basis- expansion method we decompose the solution using a complete set of functions, namely

$$
\widetilde{f}(p)=\sum_{i} a_{i} u_{i}\left(p, p_{0}\right),
$$

where the $u_{i}\left(p, p_{0}\right)$ are the eigenstates of the nonrelativistically reduced kernels (of the momentum-space Schrödinger equation), where the linear parameters $a_{i}$ and the nonlinear parameter $p_{0}$ (the inverse Bohr radius) are optimized. This leads to a standard matrixdiagonalization problem. The method gave good results for low $\alpha$ but near the critical region (i.e., where the bound-state mass vanishes) the convergence was poor, resulting in a very rapid growth in the number of basis states required. The results for this method in the trivial case of one expansion function was presented in Ref. 4.

We could, however, obtain reasonable convergence right up to the critical value with the optimized discretization method. The integral equation is solved on a lattice. The semi-infinite range is first mapped onto a finite interval. The logarithmic singularity at $p=q$, although integrable, is numerically troublesome and can be tamed with a standard trick. To this end we write

$$
\begin{aligned}
\int K(p, q) f(q) d q= & f(p) \int K(p, q) d q \\
& +\int K(p, q)[f(q)-f(p)] d q,
\end{aligned}
$$

where the first integral can be performed analytically (or numerically as the case may be).

Optimized discretization leads again to a matrix eigenvalue problem, namely

$$
\sum_{j}\left(\sum_{k} B_{i k}^{-1} A_{k j}-\frac{1}{\alpha} \delta_{i j}\right) f\left(\xi_{j}\right)=0
$$

where 


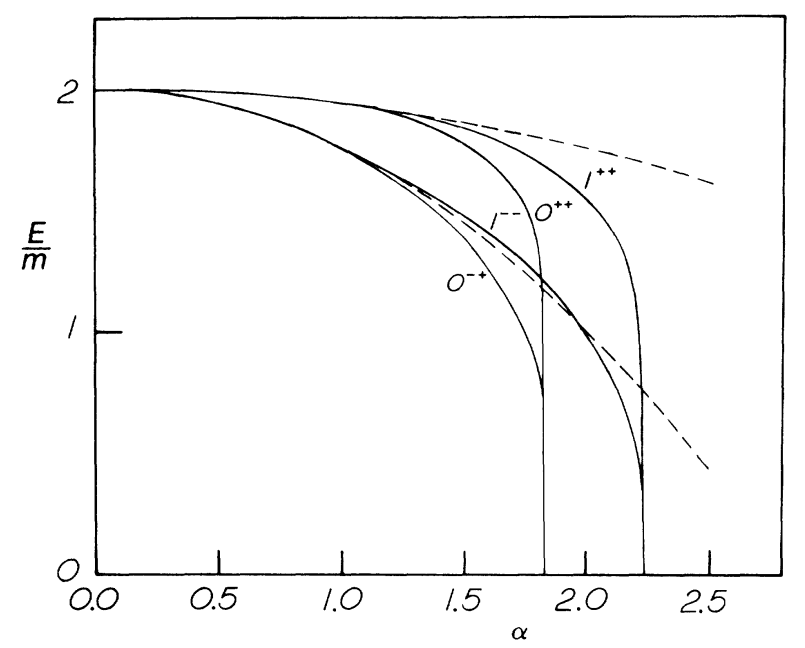

FIG. 1. Two-particle bound-state mass vs $\alpha$. The quantum numbers of the curves are indicated $0^{-+}, 1^{--}, 0^{++}$, and $1^{++}$. The dashed curves show the nonrelativistic dependence $2-\alpha^{2} / 4 n^{2}$.

$$
\begin{aligned}
& A_{i j}=\frac{1}{4 \pi}\left[W_{j} \frac{\xi_{j}}{\xi_{i}} K\left(\xi_{l}, \xi_{j}\right)\right. \\
& +\delta_{i j}\left[\int \frac{q}{\xi_{l}} K\left(\xi_{i}, q\right) d q\right. \\
& \left.\left.\quad-\sum_{k} W_{k} \frac{\xi_{k}}{\xi_{l}} K\left(\xi_{l}, \xi_{k}\right)\right]\right], \\
& B_{i j}=\delta_{i j}\left[2 E\left(\xi_{i}\right)-M\right],
\end{aligned}
$$

and

$$
K\left(\xi_{i}, \xi_{i}\right) \equiv 0
$$

The weighting coefficients $W_{i}$ and the roots $\xi_{i}$ are chosen according to the method of Gaussian quadrature.

Convergence was obtained typically to five figures with 100 points for most of the curve. At the critical point 300 points were required to obtain close to three-figure accuracy.

The results are presented in Fig. 1 where we have plotted the bound-state mass versus $\alpha$. We remind the reader of the nonrelativistic correspondence: $\left(J^{P C},{ }^{2 s+1} L_{J}\right)$, $\left(0^{-+},{ }^{1} S_{0}\right),\left(1^{--},{ }^{3} S_{1}\right),\left(1^{++},{ }^{3} P_{1}\right),\left(0^{++},{ }^{3} P_{0}\right)$, where, for the vector case, we have listed only the lowest of the two possible $L$ states. The curves at low $\alpha$ follow the $2-\alpha^{2} / 4 n^{2}$ nonrelativistic behavior. It is surprising how closely the curves follow the nonrelativistic curves for $\alpha$ 's up to $\alpha=1$. This is due to a fortuitous cancellation of the relativistic kinetic- and potential-energy corrections.

The most dramatic feature of the curves is the rapid turnaround behavior near the critical value and the degeneracy of the $0^{-+}$and $0^{++}$as well as the $1^{--}$and $1^{++}$states. Note in both cases this is a degeneracy of $\alpha_{c}$ of what was at low $\alpha 1 S$ and $2 P$ states. This can be understood in the following way. If for the moment we con- sider the massless theory the respective kernels become identical. This is a reflection of the fact that the $m=0$ theory is chirally symmetric. In the absence of spontaneous symmetry breaking one expects parity doubling in the spectrum. Furthermore, we have explicitly solved the massless integral equations and find that the critical values are $^{5}$

$$
\begin{aligned}
& \alpha_{c}\left(0^{-+}, 0^{++}\right)=\frac{4}{\pi / 2+2 / \pi} \approx 1.81, \\
& \alpha_{c}\left(1^{--}, 1^{++}\right)=\frac{8 / \sqrt{3}}{\pi \sqrt{3} / 4+4 / \sqrt{3} \pi} \approx 2.20 .
\end{aligned}
$$

In both cases $\widetilde{f}(p)=p^{-2}$.

These values are very close to our best numerical values for the massive theory:

$$
\begin{aligned}
& \alpha_{c}\left(0^{++}\right)=1.826 \\
& \alpha_{c}\left(0^{-+}\right)=1.828 \quad(\text { and scalar in SQED }), \\
& \alpha_{c}\left(1^{--}\right)=2.216 \\
& \alpha_{c}\left(1^{++}\right)=2.221
\end{aligned}
$$

The lack of rapid convergence near the critical value is the result of large cancellations between the kinetic and potential energies which just precisely balance when the wave function goes over to the massless form $\widetilde{f}(p)=p^{-2}$. This is a state which is extremely localized in configuration space sitting deep in the potential well at the origin. The energies involved are so large that a small fermion mass becomes irrelevant.

We believe that even though we have a limited Fock space and we are not sensitive to field-theoretic effects, this qualitative feature will persist. In the absence of spontaneous symmetry breaking, it will certainly remain true in the massless theory. We would find it surprising if the massive theory which joins so smoothly to the massless one at this order in Fock space would cease to do so at higher order.

To summarize and to also leave a clear impression with the reader, we would like to emphasize that although systematic improvement and enhancement of the Fock space is possible (work in this direction is in progress), ${ }^{6}$ at the present level we can only claim to have studied the relativistic two-body problem. Since the formalism is soundly based on the quantum-field-theoretic Hamiltonian and no pathologies appear (imaginary eigenvalues, etc.), we feel it is a desirable and promising technique.

\section{ACKNOWLEDGMENTS}

The authors wish to thank J. W. Darewych and Marko Horbatsch for numerous stimulating discussions. Two of us (R.K. and W.D.) would like to thank Rolf Tarrach and the Departament d'Estructura i Constituents de la Materia at the Universitat de Barcelona for their gracious hospitality at the time when this work was begun. We would like to acknowledge the financial assistance of the Natural Sciences and Engineering Research Council of Canada and Comisión Asesora Investigación Científica y Técnica, Spain. 
${ }^{1}$ L. I. Schiff, Phys. Rev. 130, 458 (1963); T. Barnes and G. I. Ghandour, Phys. Rev. D 22, 924 (1980); P. M. Stevenson, ibid. 32, 1389 (1985).

${ }^{2}$ Massless spinor and scalar QED were discussed in J. Finger, D. Horn, and J. E. Mandula, Phys. Rev. D 20, 3253 (1979).

${ }^{3}$ G. Hardekopf and J. Sucher, Phys. Rev. A 30, 703 (1984); 31, 2020 (1985).

${ }^{4}$ R. Koniuk and J. W. Darewych, Phys. Lett. B 176, 195 (1986); W. Dykshoorn, R. Koniuk, and J. W. Darewych, in Proceedings of the International Workshop on Variational Calcula- tions in Quantum Field Theory, Wangerooge, West Germany, 1987, edited by L. Polley and D. E. L. Pottinger (World Scientific, Singapore, 1988).

${ }^{5}$ The pseudoscalar massless result was obtained by J. W. Darewych and M. Horbatsch, J. Phys. B 22, 973 (1989). The value of $\alpha_{c}$ in this case was found by an asymptotic analysis of the massive theory in Ref.3.

${ }^{6}$ W. Dykshoorn and R. Koniuk, following paper, Phys. Rev. A 41, 64 (1990). 